Journal of The Magnetics Society of Japan Vol. 13, Supplement, No. S1 (1989)

(C) 1989 by The Magnetics Society of Japan

\title{
DEPENDENCE OF LOWER SIDEBAND ENHANCEMENT EFFECT ON VARIOUS RECORDING MEDIA IN VCRS
}

\author{
Masaaki KOBAYASH ${ }^{*}$, Yasutoshi YAMAMOTO ${ }^{* *}$ and Yoshitomi NAGAOKA* \\ *Development Research Laboratory, ${ }^{* *}$ HDTV Development Center, \\ Matsushita Electric Industrial Co.,LTD.,Kadomashi, Osaka 571, Japan
}

\begin{abstract}
The lower sidebands are enhanced on a Co- $\not \mathrm{Fe}_{2} \mathrm{O}_{3}$ tape when AM signals and Dual-frequency signals are recorded. The order of enhancement magnitude is AM> Dual-frequency $>\mathrm{FM}$ on a Co-rFe $\mathrm{O}_{3}$ tape. On the other hand, the lower sidebands are not enhanced on a ME tape. These phenomena are observed with actual examinations and computer simulations including perpendicular mode. Compared with the results of experiments and simulations, it is conclude that the lower sidebands enhancement depends on tape thickness.
\end{abstract}

\section{INTRODUCTION}

It is well known that lower sidebands of frequency-modulated signals are enhanced by the recording and reproducing process [1]. This phenomenon is called lower sidebands enhancement and is important for analog VCR design. The phenomenon on perpendicular media were studied recently [2], [3].

We had defined that the lower sidebands enhancement on pigment tapes depends on signal modulation methods [4].

In this paper, dependence of lower sidebands enhancement on recording media was studied by being compared with actual examinations and computer simulations including perpendicular mode.

\section{EXPERIMENTS WITH A VCR}

To study the dependence of lower sidebands enhancement, a $\mathrm{Co}-\gamma \mathrm{Fe}_{2} \mathrm{O}_{3}$ tape ( for S-VHS) is selected out of pigment media and a metal evaporated ( ME ) tape is selected out of metal thin film media. A VCR for consumer use is modified for this experiment. Recording and repro- ducing amplifiers are tuned to have flat frequency response. Scanning speed of video heads is $5.8 \mathrm{~m} / \mathrm{sec}$.

\subsection{Recording of continuous waves}

Continuous waves are recorded on the media. Fig.l shows reproduced signal levels. The recording currents are set to reproduce the maximum level at each frequency, which is called the optimum recording currents.

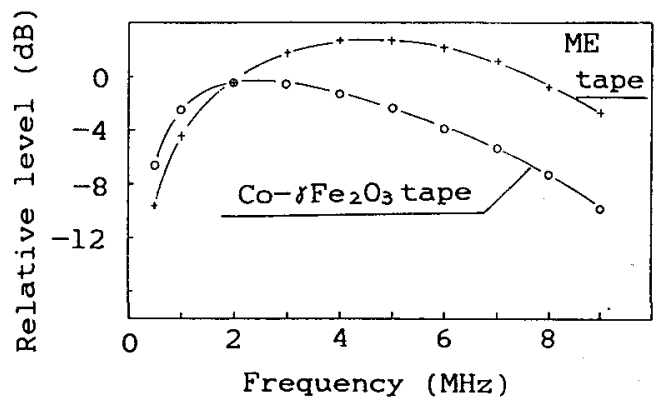

Fig.1 Reproduced signal levels. 


\subsection{Recording of modulated signals}

Three kinds of modulated signals ( AM, Duel-frequency, FM with low modulation index ) are applied to recording with the VCR. These recording signals are
a) $\mathrm{AM}$
$\left(J_{-1}, J_{0}, J_{+1}\right)$
b) Duar
$\left(J_{-1}, J_{0}\right)$,
c) FM $\left(J_{-1}, J_{0}, J_{+1}\right)$.

The spectra components of the signals are shown in ( ). The $J_{-1}$ denotes a lower sideband, the Jo denotes a carrier; and the $J_{+1}$ denotes a upper sideband.

For example, $J_{-1}=1.45 \mathrm{MHz}$

$$
J_{0}=6.45 \mathrm{MHz}
$$$$
\mathrm{J}_{41}=11.45 \mathrm{MHz} \text {. }
$$

The recording current of $J_{0}$ is set the optimum recording current and the recording current of $J_{-1}$ is set $-12 \mathrm{aB} \mathrm{com}^{-}$ pared with the recording current of $J_{0}$.

The levels of reproduced spectra out of the $\mathrm{Co}-\gamma \mathrm{Fe}_{2} \mathrm{O}_{3}$ tape are shown in $\mathrm{Fig.-}$ 2(a). In Fig. 2 and 6 , the fine lines and doted lines are reference lines which indicate the reproduced $J_{0}$ level of $F M$ recording and $-12 \mathrm{~dB}$ level respectively. It is recognized that the reproduced Jo level of the Dual-frequency recording little decreases compared with the reproduced Jo level of FM recording, and the reproduced $J_{-1}$ level of the Dualfrequency recording is 4 to $5 \mathrm{~dB}$ greater than that of the FM recording, and the reproduced Jo level of the $A M$ recording is about $2 \mathrm{~dB}$ smaller than that of the FM recording, and the reproduced $J_{-1}$ level of the $A M$ recording is about $7 \mathrm{~dB}$ greater than that of the FM recording.

The level of reproduced spectra out of the ME tape are shown in Fig.2(b). It is recognized that the reproduced $J_{-1}$ levels of the three kinds of recording are nearly equal and are lower than $-12 \mathrm{~dB}$ respectively.

The results shows as follows.

1) The lower sidebands are enhanced on a $\mathrm{Co}-\gamma \mathrm{Fe}_{2} \mathrm{O}_{3}$ tape when $\mathrm{AM}$ signal and Dual-frequency signal are recorded.

2) The order of enhancement magnitude is AM > Dual-frequency $>\mathrm{FM}$ on a $\mathrm{Co}-\mathrm{FFe}_{2} \mathrm{O}_{3}$ tape.
3) The lower sidebands enhancement does not occur on a ME Tape.

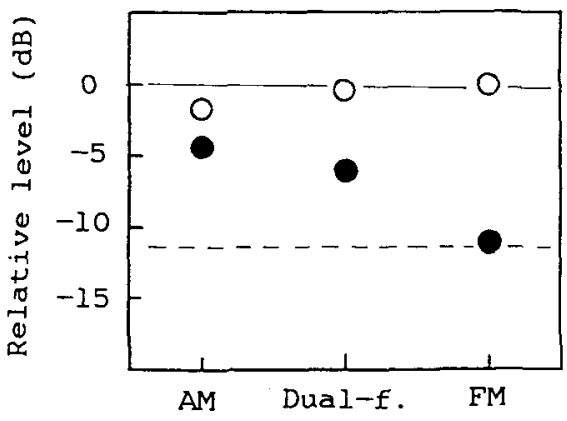

(a) $\mathrm{Co}-\gamma \mathrm{Fe}_{2} \mathrm{O}_{3}$ tape.

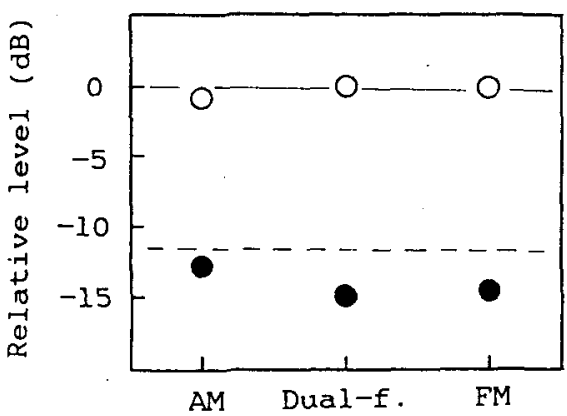

(b) ME tape.

OdB : reproduced Jo Ievel of FM

0 : Jo ( $6.45 \mathrm{MHz})$

- $J_{-1}(1.45 \mathrm{MHz})$

Fig. 2 Levels of reproduced spectra.

\section{Computer simulation}

To explain the lower sidebands enhancement effect, computer simulations are used and the simulation results are compared with the experiment results shown in section two. Longitudinal magnetization and perpendicular magnetization are applied to the simulation respectively. Magnetic media is separated into multiple thin layers as shown in Fig.3. The residual magnetization is determind every thin layer by a hysteresis-loop tracing method. The method consists of a hystertesis-loop model shown in Fig.4. and a Karlquist's 
head-field distribution model. On reproducing, reciprocal theorem is used as means to read out signals from the every thin layer. Since the simulations do not include self-demagnetization and vector magnetization, it is considered that the results of them have qualitative meanings. The conditions for simulations is shown in Table 1 .

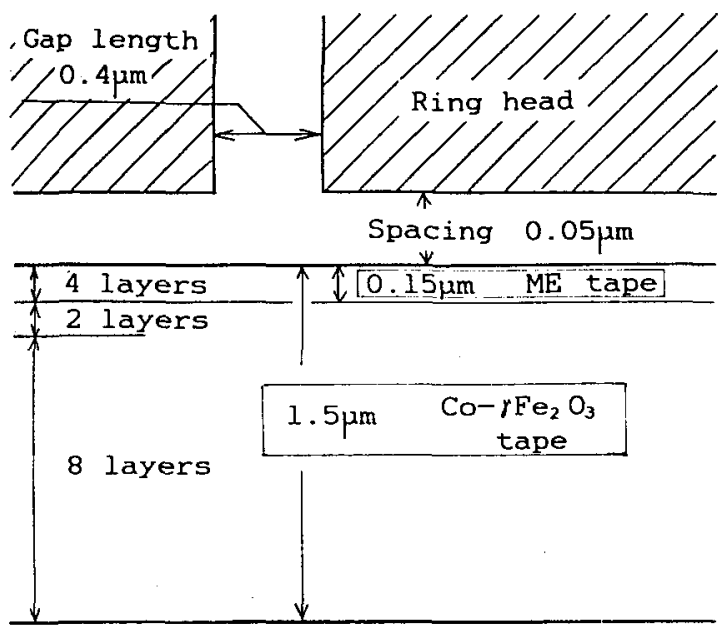

Fig. 3 The tapes and head model for simulations.

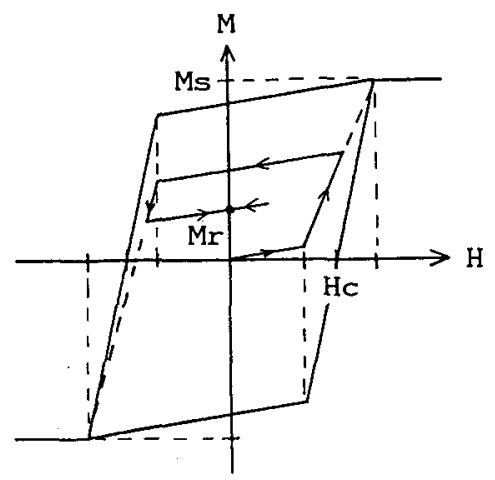

Fig. 4 Rectilinear hysteresis-loop model.
Table 1 Conditions for simulations

\begin{tabular}{|c|c|c|c|}
\hline & $\mathrm{Co}-\gamma \mathrm{Fe}_{2} \mathrm{O}_{3}$ & \multicolumn{2}{|c|}{ ME } \\
\hline mode & $/ 1$ & $/ /$ & $\perp$ \\
\hline $\mathrm{Hc}(\mathrm{Oe})$ & 850 & 1000 & 1400 \\
\hline Br(Gauss) & 2100 & 3500 & 1300 \\
\hline $\mathrm{s}$ & 0.8 & 0.7 & 0.28 \\
\hline $\begin{array}{l}\text { thickness of } \\
\text { media }(\mu \mathrm{m})\end{array}$ & 1.5 & 0.15 & 0.15 \\
\hline $\begin{array}{l}\text { head gap- } \\
\text { length }(\mu \mathrm{m})\end{array}$ & 0.4 & 0.4 & 0.4 \\
\hline $\begin{array}{l}\text { head-tape } \\
\text { space }(\mu \mathrm{m})\end{array}$ & 0.05 & 0.05 & 0.05 \\
\hline
\end{tabular}

\subsection{Recording of continuous waves}

Continuous waves are recorded with the computer simulations. Fig.5 shows reproduced signal levels which are obtained by the longitudinal mode computer simulation.

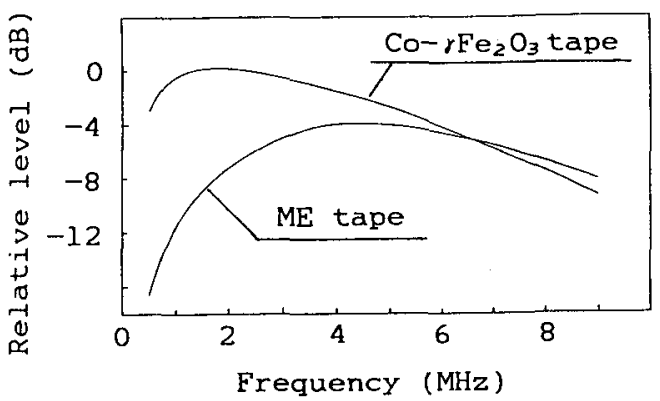

Fig.5 Reproduced signal levels.

Compared with Fig.l and Fig.5, it is said that the frequency characteristics of $\mathrm{Co}-\gamma \mathrm{Fe}_{2} \mathrm{O}_{3}$ tape and $\mathrm{ME}$ tape are similer respectivel.y. On the ME tape, reproduced levels with simulation are lower than reprodiced levels with actual experiments, even if perpendicular reproduced levels shown in Fig.7 were added to the leveis. 


\subsection{Recording of modulated signals}

The three kinds of modulated signals are recorded and reproduced with computer simulations. The reproduced signal levels are shown in Fig.6. Longitudinal components are only used in the simulations.

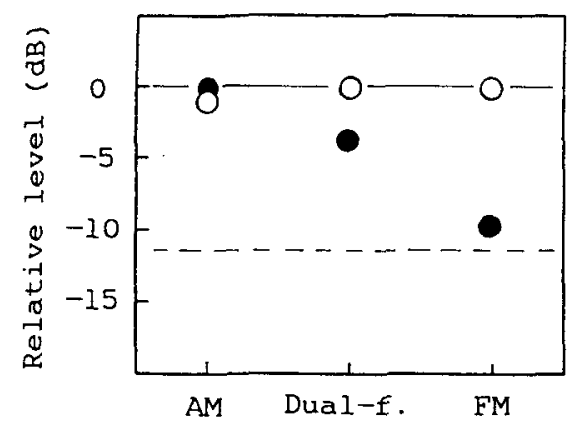

a) $\mathrm{Co}-\gamma \mathrm{Fe}_{2} \mathrm{O}_{3}$ tape.

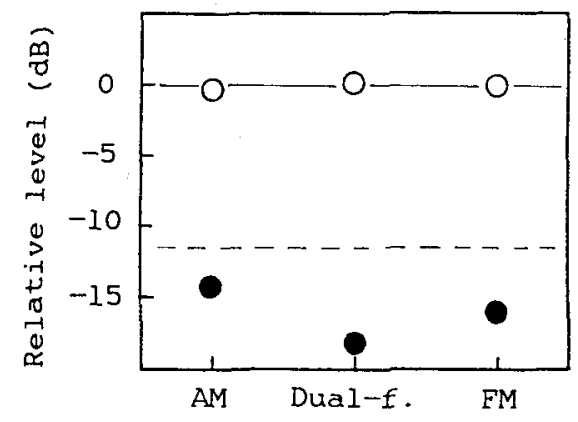

b) $\mathrm{ME}$ tape.

$\mathrm{OdB}$ : reproduced Jo level of FM $O$ : Jo $(6.45 \mathrm{MHz})$

: $J_{-1}(1.45 \mathrm{MHz})$

Fig.6 Levels of reproduced spectra

Compared with Fig.2 and Fig.6, it is said that reproduced levels with simulations are similar that with actual experiment on the each tape.

\subsection{Perpendicular simulation}

Since the ME tape has some perpendicular components as shown in Table I, perpendicular simulations are applied to the ME tape. The simulation methods are adopted the same method as longitudinal simulations, but it is different points that perpendicular components are adopted as magnetization and head-field. Fig.7(a) shows results of perpendicular simulations and Fig.7(b) shows results of perpendicular simulations compensated demagnization.

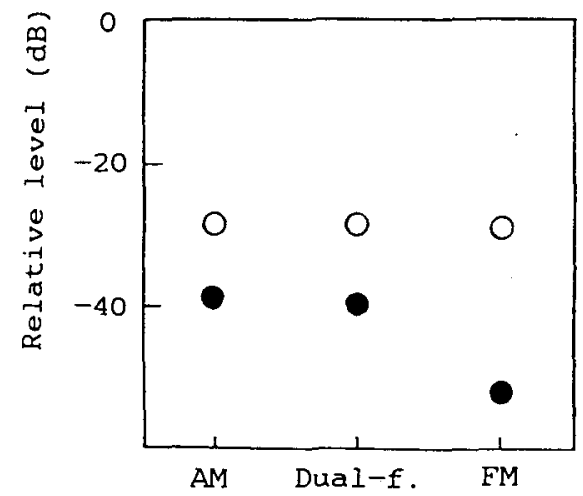

(a) Without demagnetization compensation.

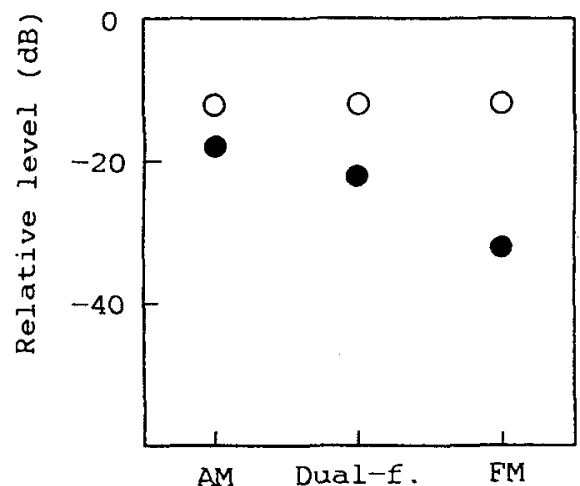

(b) With demagnetization compensation. OdB : reproduced Jo level of the FM ( longitudinal mode)

0 : Jo $(6.45 \mathrm{MHz})$

Fig. 7 Perpendicular simulations on ME tape. 


\section{CONSIDERATION}

The lower sidebands are enhanced on a $\mathrm{Co}-\mathrm{Fe}_{2} \mathrm{O}_{3}$ tape when $\mathrm{AM}$ signal and Dual-frequency signal are recorded. The order of enhancement magnitude is $\mathrm{AM}>$ Dual-frequency $>\mathrm{FM}$ on $\mathrm{Co}-\mathrm{Fe}_{2} \mathrm{O}_{3}$

tape. But, the lower sidebands are not enhanced on a ME tape. These phenomena are observed with actual examinations and computer simulations.

The reasons of enhancement or no enhancement are considered as follows.

1) $\mathrm{A} \mathrm{Co}-\gamma \mathrm{Fe}_{2} \mathrm{O}_{3}$ tape has enough thickness to record $1 / 4$ of recording wave length.

2) An upper sideband of AM enhances envelope fluctuation of a recording wave and an upper sideband of FM rolis envelope fluctuation of that. And, as Dual-frequency has not upper sideband, envelope fluctuation of that is midway between $A M$ and FM. The frequency of envelope fractuation is $J_{-1}$. For that reasons, the order of the lower sidebands enhancement is in proportion to the fluctuation.

3) A ME tape has not enough thickness to record $1 / 4$ of recording wave length, so that the thickness operates like a limiter.

4) Therefore, the lower sidebands enhancement is not observed on a ME tape with actual experiments and longitudinal and perpendicular simulations.

\section{CONCLUSIONS}

Compared with the results of actual experiments and simulations, It is defined that the lower sidebands enhancement depends on tape thickness. In the case of thick media such as a $\mathrm{Co}-\gamma \mathrm{Fe}_{2} \mathrm{O}_{3}$ tape, the lower sidebands enhancement depends on kind of signal modulation method.

\section{Acknowledgement}

\begin{abstract}
The authers wish to express their sincere gratitude to Dr.M.Nagasawa, Directer of Development Research Laboratory of Matsushita Electric Industrial Co., Ltd. for permission to publish this paper, and thank for Mr.K.Sinohara, manager of Video Recorder Division of Matsushita Electric Industrial Co.,Ltd. to supply them with a ME tape.
\end{abstract}

\section{Reference}

[1] K. Yokoyama, "Basic Studies of Video Tape Recorder Design", NHK Technical Journal, 21, 4 (1969).

[2] J.Numazawa, Y.Yoneda, S.Nakagawa, "Investigation of Video Recording Methods by Perpendicular Magnetic Recording" IECE Tech. Report on Magnetic Recording, MR84-11 (1984).

[3] T.Ozue, I.Saito, K.Takahashi, T.Narahara, K.Kawazoe, J.Hokkyo, "High density Video Recording by Perpendicular Magnetic Recording", IECE Tech. Report on Magnetic Recording,MR84-53 (1985). [4] M.Kobayashi, Y.Yamamoto, S.AKI, Y.Nagaoka, "A Study of Lower Sideband Enhancement on Analog VCR", The Trans. of the IEICE,E72, 4,pp326-327 (1989). 